\title{
Changes in the expressions of thiamine biosynthesis genes (THIC and THI1/THI4) in oil palm (Elaies guineensis) as response to salinity stress
}

\begin{abstract}
Thiamine or also known as vitamin B 1 , it is the first water soluble B-complex vitamin to be identified which plays an important role as both cofactor and non-cofactor. Its active form, thiamine pyrophosphate (TPP) is important as a cofactor for various types of enzymes involved in central metabolism. Besides that, thiamine has been shown to have a role in plant protection against stress. In this study, we investigated the expressions of the first two enzymes involved in thiamine biosynthesis pathway, THIC and THI1/THI4 when subjected to salinity stress induced by sodium chloride $(\mathrm{NaCl})$ at various concentrations, namely, 0,50 , 150 and $200 \mathrm{mM}$. RT-PCR was conducted to amplify both gene transcripts and the intensities of the bands were analyzed by using the ImageJ software. Overall, there was an increase in expression for both THIC and THI1/THI4 gene transcripts upon stress application. For THIC, the highest level of expression was observed on day 7 for $50 \mathrm{mM} \mathrm{NaCl}$ treated oil palm seedling with an increase of up to $331 \%$, while for THI1/THI4, the highest level of expression was $723 \%$ on day 3 when $200 \mathrm{mM} \mathrm{NaCl}$ was applied to the oil palm seedlings. These results obtained are in agreement with suggestions that thiamine plays an additional role in plant protection against stress as it may lead to an overexpression of thiamine in general.
\end{abstract}

Keyword: Abiotic stress; Gene expression; Oil palm; Salinity stress; Thiamine; Vitamin B1 